\title{
COUPLING COMPUTATION OF THE FLOW FIELD AND THE LARGE DEFORMATION OF MEMBRANE STRUCTURE OF STRATOSPHERE AIRSHIPS
}

\author{
Ph. D. Candidate: LIU Jian-min \\ Department of Engineering Mechanics, Shanghai Jiaotong University, Shanghai 200240, China, \\ E-mail: liujm501@gmail.com
}

\section{Supervisor:}

Lu Chuan-jing (Shanghai Jiaotong University) Xue Lei-ping (Shanghai Jiaotong University)

Members of dissertation defense committee: Dai Shi-qiang (Shanghai University) Chairman Zhu De-xiang (CSSRC) Zhang Hui-sheng (Fudan University) He You-sheng (Shanghai Jiaotong University) Miao Guo-ping (Shanghai Jiaotong University) Lu Chuan-jing (Shanghai Jiaotong University) Xue Lei-ping (Shanghai Jiaotong University)

Time of dissertation defense: April 2, 2008

\begin{abstract}
In this article, the mathematical model of the coupling of the three-dimensional fluid flow and the large deformation of membrane structure is established. The fluid-structure coupling interaction is simulated using the computational codes developed by the authors. By analyzing the interactions of membrane and flow field, the aeroelasticity of the airship is detailed. All the results are adopted in the focused study of the stratosphere airship in trimmed state.
\end{abstract}

Key words: three-dimensional flow, large deformation of membrane structure, fluid-structure coupling, aeroelastics, airships

Brief introduction: This article focuses on the coupling of the deformation of the airship in the stratosphere and the peripheral flow field around. The main contents are as follows:
Firstly, in the study, the mathematical models and the numerical solvers of the coupling computation of three-dimensional fluid flow and the large deformation of membrane are established respectively. The pressure correction method SIMPLE, which is based on the finite volume method, is proposed for the numerical simulation of the fluid flow. For the numerical simulation of membrane deformation, the nonlinear finite element method is employed.

Secondly, by establishing the close coupled computation solution for the analysis of membrane in three-dimensional flow, the problems of grids deformation and interface information exchanges in the fluid-structure coupling computation have been solved.

Thirdly, the computational codes of coupled computation are programmed. The program modules of fluid analysis, structure analysis, grid generation, and interface information exchange technique are integrated.

Fourthly, with the fluid-structure coupling approach, the aeroelasticity of the stratosphere airship in the trimmed condition is specialized. Different computation models and flow conditions are compared to discuss the aeroelasticity of the bare hull and the hull-fin configuration, respectively. All the results are helpful for establishing the numerical computation and analysis tool for the design of the stratosphere airship in terms of aerodynamics, dynamics, structure, and control system. 